\title{
PVCM: Assisting multi-hop communication in vehicular networks using parked vehicles
}

\author{
Josip Balen, Goran Martinovic \\ Faculty of Electrical Engineering \\ J. J. Strossmayer University of Osijek \\ Osijek, Croatia \\ Email: firstname.lastname@etfos.hr
}

\author{
Koosha Paridel, Yolande Berbers \\ Department of Computer Science \\ KU Leuven \\ Leuven, Belgium \\ Email: firstname.lastname@cs.kuleuven.be
}

\begin{abstract}
Multi-hop communication in vehicular ad hoc networks is a challenging task due to the dynamic and unbalanced nature of these networks. In the suburban areas or during the night when the density of vehicles is low, multi-hop communication is only possible if (i) the vehicles store-carry-forward the messages or (ii) by using roadside units. Each of the solutions has its drawback. The former introduces delivery delay and the latter is an expensive solution. In this paper, we propose the Parked Vehicle Communication Model (PVCM) to use the parked vehicles along the roads to act as relay nodes and assist the communication in low density situations. We argue that the number and the distribution of parked vehicles is suitable to serve our purpose. We also show that the automotive battery can be utilized to keep the on-board unit running without draining all its energy.
\end{abstract}

\section{INTRODUCTION}

Vehicular ad hoc networks (VANETs) have gained attention in the recent years due to their use in road safety and other commercial applications. VANETs are networks of vehicles equipped with wireless devices that are interconnected and can exchange information. Moreover, the topologies in VANETs are very dynamic and non-uniformly distributed. As more vehicles are getting equipped with wireless devices, we expect to have large-scale VANETs in near future.

In our current work [1], we present a large-scale contextbased data dissemination system in VANETs that exploits the contextual information of the vehicles in order to provide an efficient multi-hop communication protocol between vehicles. Our system solely relies on the ad hoc network of vehicles and does not assume any sort of infrastructure or roadside units.

The density of the network is an important factor in communication in VANETs. In order to perform multi-hop communication in timely fashion, the network of vehicle should have a certain amount of density. The minimum level of density required for communication is dependent on the wireless communication range, and varies in different environments. For example, in a highway with a long line of sight, the wireless communication range is longer, but in an urban environment with buildings acting as obstacles, the wireless communication range is shorter. Real life experiments show that wireless communication between vehicles can fail even at distance of $30 \mathrm{~m}$ due to obstacles in urban environments [2]. In addition to obstacles and environment effects, low density can happen in suburban areas with low traffic, during the night and off-peak hours, and due to the low penetration in the initial stages of deployment of wireless On-Board Units (OBUs) in vehicles.

There are two approaches to overcome the problems caused by low density:

- Store-carry-forward model: In this model, vehicles store the received messages and carry them for a certain amount of time, and upon contact, they share the stored messages with other vehicles. This model does not need any additional infrastructure, but increases the delay of message delivery.

- Roadside units: In this approach, roadside units are installed along the road, and vehicles can send and receive messages from them. The roadside units are usually connected with each other and therefore, the message delivery delay is low. However, installing roadside units comes with a considerable expense for infrastructure.

However, there is a possibility to use the parked vehicles along the roads as relay nodes to address the problem of communication in sparse networks. When vehicles are parked, we can still use the OBU to participate in the communication. Parked vehicles represent static roadside nodes characterized by large number, long-time staying, wide distribution and specific location [3]. We argue later in the paper that considering the energy consumption of the OBU and the average capacity of the battery inside the vehicles, the energy stored in the batteries is enough to keep the OBU in operation for many hours without draining the battery. In this paper we propose the Parked Vehicle Communication Model (PVCM) to use parked vehicles in order to assist our communication protocol by relaying messages: (i) during night and off-peak hours, (ii) in the suburban areas, (iii) around the obstacles and city blocks, (iv) after the initial deployment of communication devices in vehicles, and (v) in underground garages.

The rest of this paper is organized as follows: Section II discusses the related work. In Section III we present the idea of using parked vehicles as relay nodes to improve the communication. Section IV argues about the feasibility of using vehicle batteries as a source of energy for OBU. In Section V, we conclude the paper and discuss the future work. 


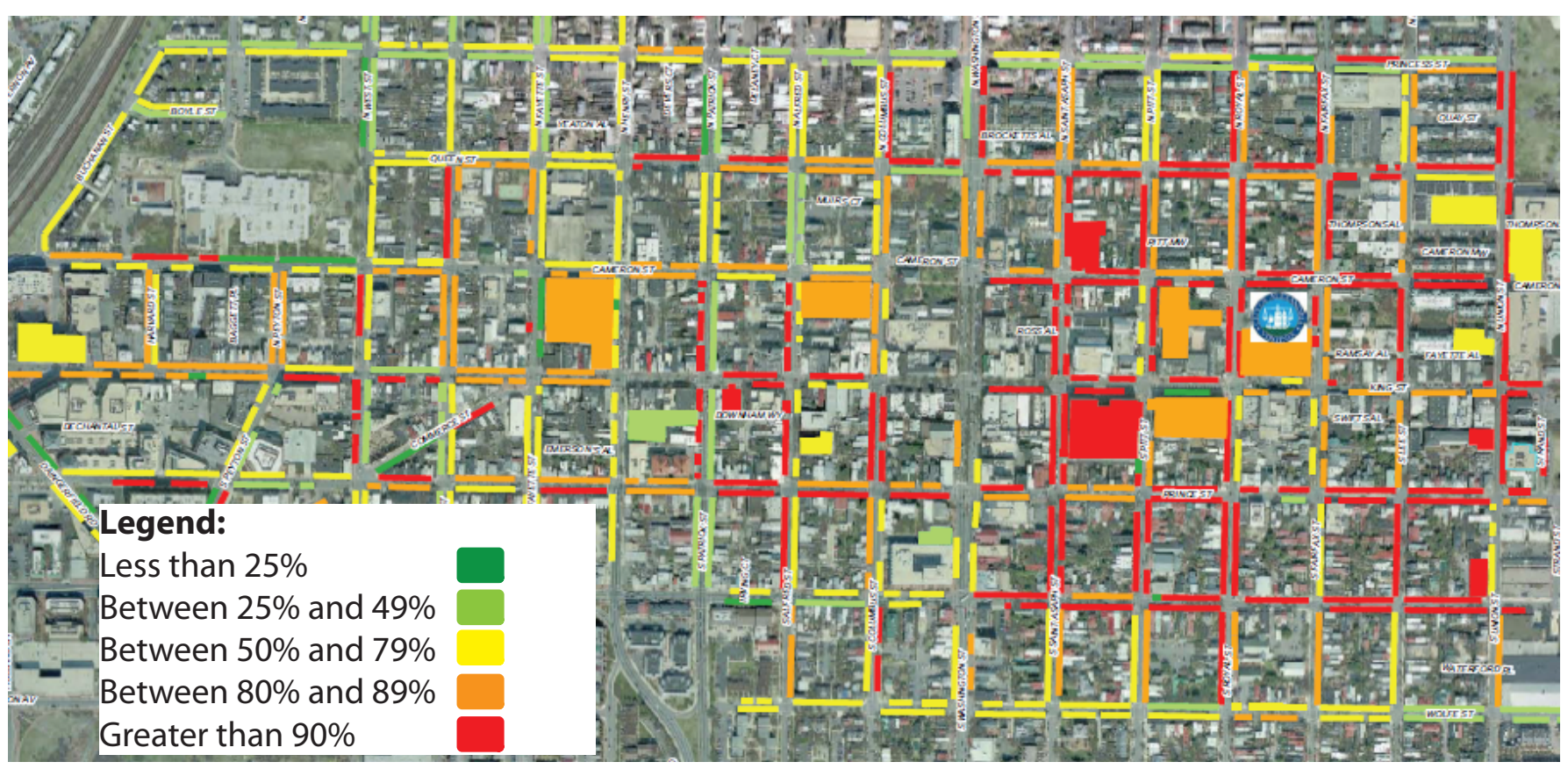

Fig. 1: Parking Utilization During Weekday Afternoon in Old Town Alexandria.

\section{RELATED WORK}

In [3] authors propose the Parked Vehicle Assistance (PVA), which allows parked vehicles to join VANETs as static nodes. They calculation and realistic simulation results show that using even small proportion of parked vehicles (10\% and $30 \%$ ) can greatly improve connection ratio and duration, and dramatically lower the period during which two vehicles are disconnected.

In [4] authors describe in detail vehicular content distribution using parked vehicles called ParkCast. Parked vehicles are first grouped into per-street line cluster with cluster heads that are able to manage all parked vehicles and their resources, and arrange content distribution. Simulation results show that ParkCast dramatically reduces downloading delay and improves downloading rate of large content distribution, speeds up message dissemination of small messages, specially in sparse traffic conditions and efficiently supports empty car downloading so vehicle are always provided with up to date information. However, they assume using additional rechargeable batteries for OBUs that are not necessary since vehicles already have high capacity batteries and power consumption used for communication between vehicles is not high.

In [2] authors propose to use parked vehicles as relay nodes in order to connect two vehicles that are located at two sides of an obstacle or a building. They use parked vehicles only to communicate the periodically emitted Cooperative Awareness Messages (CAMs) that contain information such as position and speed. CAM messages are used by other vehicles in the vicinity to know about the presence of a nearby vehicle. These messages are only propagated up to two hops. The authors examine how much the usage of parked vehicles can increase the safety of nearby vehicles, but they do not propose any specific relay strategy. They evaluate their idea by simulating a synthetic manhattan grid scenario and a realistic suburban scenario, and they conclude that by using parked vehicles, the amount of vehicles that can be reached by CAMs increases significantly that in turn, increase the driving safety.

In [5] authors present a content downloading system in vehicular networks using parked vehicles. Their system is designed for sharing big pieces of data between vehicles and exchanging data only with on-hop communication. They suggest to use parked vehicles instead of roadside units to lower the costs of such a system. Their goal is to maximize content freshness and utilize the radio resources. They evaluate their system using simulation in a scenario from the city of Ingolstadt, with 963 moving vehicles and 80 parked vehicles. They measure network traffic and content freshness by the percentage of parked vehicles, and claim that they by using parked vehicles, they can increase content freshness and radio resource utilization.

\section{USING PARKED VEHICLES FOR IMPROVING COMMUNICATION}

\section{A. Parking in urban areas}

As an example of parking utilization in urban areas we took the parking study conducted in Old Town Alexandria [6]. The study area consisted of approximately 85 city blocks with 8332 publicly accessible parking spaces. On-street parking accounts for the majority of available parking and comprises 53\% of the system. Publicly accessible parking garages account for $42 \%$ of the inventory and surface lots contain $5 \%$ of the parking system. The parking counts were conducted during the different six time periods during weekdays and weekend and the average parking space occupancy was $62.5 \%$. On Figure 1 


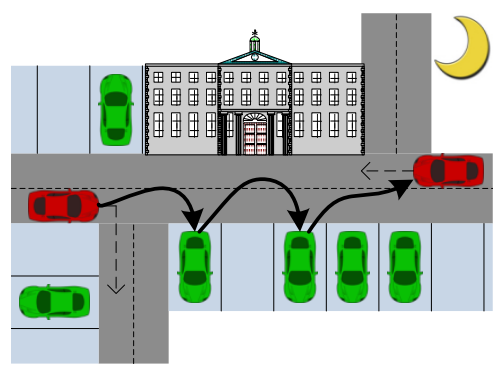

(a) During night and off-peak hours.

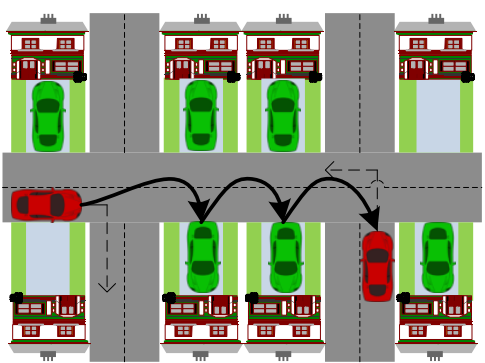

(b) Suburban area.

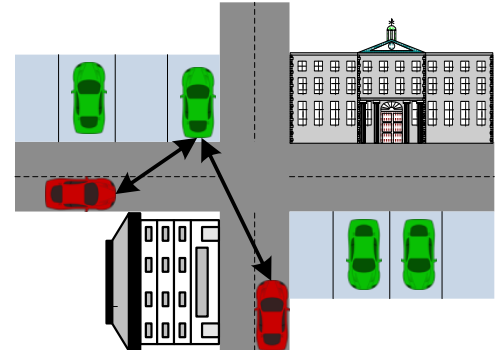

(c) Shadowing caused by obstacles.

Fig. 2: Typical scenarios where PVCM can be used for improving communication.

the parking utilization during weekday afternoon is shown. Following conclusions can be drawn:

- Parking spaces are regularly and efficiently distributed throughout the Old Town.

- Most streets have parking spaces on both sides.

- Parking space utilization in most streets is over 50\%.

- On area approximately $1600 \mathrm{mx} 800 \mathrm{~m}$ there are average 5000 parked vehicles, which indicate very high density of parked vehicles.

However, the study does not count the vehicles in private parking spaces inside private properties that can increase the total number of parked vehicles significantly.

As the statistics suggest, there is a considerable number of vehicles parked along the roads in urban areas, throughout the whole day, and during the whole week. This shows an opportunity to utilize the parked vehicles to assist inter-vehicle communication.

\section{B. Improving communication}

Communication between parked and moving vehicles represents a hybrid communication of Vehicle-to-Vehicle (V2V) communication and Vehicle-to-Infrastructure (V2I) communication since parked vehicles represent static nodes in $\mathrm{V} 2 \mathrm{~V}$ communication. VANETs are characterized as highly dynamic network due to the constant and rapid movement of vehicles. Therefore, communication in VANETs is mostly affected with disruptions and delays as a result of network disconnections and partitions. Parked vehicles show several advantages when compared to the moving vehicles. They remain static for longer period and mostly they exist in large numbers. Parking spaces are mostly efficiently distributed throughout the urban areas and parked vehicles are wide distributed at pre-known locations.

Therefore, we propose to use parked vehicles in order to assist our communication protocol by relaying messages in the following scenarios:

- During night and off-peak hours - the traffic density is very low and most vehicles are parked. Therefore, parked vehicles can be used to enable information relaying between moving vehicles that are out of the communication range, as shown in Figure 2a.

- In the suburban areas - mostly consists of low capacity roads and lots of vehicles are parked in front of the houses on alleyways. These vehicles can be efficiently used for relaying information and improving communication range, as shown in Figure $2 b$.

- Around the obstacles and city blocks - due to the shadowing caused by obstacles in urban areas, communication can fail even between vehicles that are very close to each other. Therefore, parked vehicles can be used for bypassing the obstacles, as shown in Figure 2c.

- After the initial deployment of communication devices in vehicles - it will take around 15 years before all vehicles on roads are equipped [7]. At the initial phase a low number of vehicles will participate in VANET and parked vehicles would be very useful for improving the communication.

- In underground garages - vehicles in underground garages cannot receive information from the vehicles on streets due to the network disconnection. When a vehicle enters the garage it can disseminate useful information to all vehicles inside. Therefore, drivers of the vehicles that are exiting the garage in a short time have fresh information and can make driving decisions even before starting the vehicle. Furthermore, entering vehicle can receive useful information from vehicles inside the garage (i.e. free parking space).

\section{Discussion}

The main benefit of the PVCM is that it can improve connection in urban areas specially in scenarios with sparse traffic and obstacles, as stated above. Generally, traffic is unbalanced most of the time and PVCM can be used to overcome this phenomena. Roadside infrastructure is expensive and therefore, parked vehicles can dramatically lower the VANET implementation expenses in urban areas. Since parked vehicles are not stable roadside units, they cannot completely replace the infrastructure in urban areas. However, they can represent the infrastructure to some extent or provide additional support to the infrastructure. PVCM implementation on highways and open roads is difficult task since there is a small number of parked vehicles and they are mostly very distant and thus, they are out of each other's communication range. PVCM in combination with roadside infrastructure and store-carry-forward model has great potential to enable full coverage in all environments for VANETs. 


\section{CAR BATTERY USAGE}

In [8] authors tested the relationship between transmission power and packet reception performance for the V2V safety communication systems in the Dedicated Short Range Communication (DSRC) band at $5.9 \mathrm{GHz}$. The packet size in all tests was 400 bytes, the transfer rates were $3 \mathrm{Mbps}$ and $6 \mathrm{Mbps}$ and power tests were conducted in 16 different environments. They concluded that with higher transmission power of 33 $\mathrm{dBm}(\approx 2.0 \mathrm{~W})$ in comparison with $20 \mathrm{dBm}$ better performance (e.g., lower PER (Packet Error Rate) at a given distance and/or larger achievable communication range) could be achieved in most scenarios.

Sice a typical car battery has a capacity of $80 \mathrm{Ah}$ at $12 \mathrm{~V}$, and the typical $\mathrm{OBU}(\mathrm{DC} 12 \mathrm{~V}, 0.5 \mathrm{~A})$ consumes approximately $2.0 \mathrm{~W}$ of power for the communication and $4.0 \mathrm{~W}$ for the computation we can calculate the time after which the battery is exhausted as:

$$
\frac{80 \mathrm{Ah} * 12 \mathrm{~V}}{2.0 \mathrm{~W}+4.0 \mathrm{~W}}=160 \mathrm{~h}
$$

However, PVCM must not drain the battery of parked vehicle. Therefore, PVCM must turn of the OBU after the vehicle battery is spent seriously (from $30-50 \%$, communication time $\approx 48-80 \mathrm{~h}$ ) or a wake-up mechanism can be implemented. Since communication time of parked vehicles based on using existing car batteries is very long, our solution does not assume usage of additional rechargeable batteries for OBU.

\section{CONCLUSION AND FUTURE WORK}

Parked vehicles have a high degree of temporal and spatial regularity that enables higher optimizations and better resource utilization than with moving vehicles. We proposed to use parked vehicles as relay nodes to assist communication between vehicles in sparse networks, such as in suburban areas, or during the night. We discussed that on the basis of the number and the distribution of the parked vehicles in urban areas, they can play an important role in the communication. As our future work, we plan to examine the efficiency of parked vehicle utilization in communication in VANETs using simulation.

\section{REFERENCES}

[1] K. Paridel, T. Mantadelis, A.-U.-H. Yasar, D. Preuveneers, G. Janssens, Y. Vanrompay, and Y. Berbers, "Analyzing the efficiency of context-based grouping on collaboration in vanets with largescale simulation," Journal of Ambient Intelligence and Humanized Computing, vol. 3, pp. 1-16, May 2012. [Online]. Available: https://lirias.kuleuven.be/handle/123456789/350308

[2] D. Eckhoff, C. Sommer, R. German, and F. Dressler, "Cooperative awareness at low vehicle densities: How parked cars can help see through buildings," in Global Telecommunications Conference (GLOBECOM 2011), 2011 IEEE. IEEE, 2011, pp. 1-6.

[3] N. Liu, M. Liu, W. Lou, G. Chen, and J. Cao, "Pva in vanets: Stopped cars are not silent," in INFOCOM, 2011 Proceedings IEEE, april 2011, pp. $431-435$.

[4] N. Liu, M. Liu, G. Chen, and J. Cao, "The sharing at roadside: Vehicular content distribution using parked vehicles," in INFOCOM, 2012 Proceedings IEEE, march 2012, pp. 2641 -2645.

[5] F. Malandrino, C. Casetti, C. Chiasserini, C. Sommer, and F. Dressler, "Content downloading in vehicular networks: Bringing parked cars into the picture."

[6] Kimley-Horn and Associates, "Old town area parking study, final report," February 2010., available: http://www.alexandriava.gov/uploadedFiles/localmotion/ExistingParking Conditions.pdf.

[7] F. Bai and B. Krishnamachari, "Exploiting the wisdom of the crowd: localized, distributed information-centric vanets," IEEE Communications Magazine, vol. 48, no. 5, pp. 138-146, 2010.

[8] F. Ahmed-Zaid, F. Bai, S. Bai, and et al., "Vehicle safety communications - applications (vsc-a) final report: Appendix volume 2 communications and positioning," September 2011, available: http://www.nhtsa.gov/DOT/NHTSA/NVS/Crash\%20Avoidance/Technical $\% 20$ Publications/2011/811492C.pdf. 